Pacific Journal of Mathematics

SOME CONNECTIONS BETWEEN CONTINUED FRACTIONS 


\title{
SOME CONNECTIONS BETWEEN CONTINUED FRACTIONS AND CONVEX SETS
}

\author{
Robert Seall and Marion Wetzel
}

The purpose of this paper is to develop certain connections between the continued fraction solutions and the convex set solutions to some of the moment problems. In particular, we shall develop some relations between the work of Wall [3], [4] on continued fractions and the work of Karlin and Shapley [1] on convex sets. The paper is divided into two parts:

I. Stieltjes-type continued fractions and convex sets.

II. Jacobi-type continued fractions and convex sets.

Two characterizations of the moment problem for the interval $(0,1)$, one by Riesz [2] in terms of convex closures and one in term of Hankel forms, are well known. The work of Karlin and Shapley [1] shows the equivalence of these two characterizations. A third characterization in terms of a Stieltjes-type continued fraction has been given by Wall [3], [4]. In part I we give an interpretation of the parameters in this continued fraction in terms of "distances" in certain convex bodies. This interpretation, through the work of Karlin and Shapley, immediately shows the equivalence of all three characterizations.

Solutions of the moment problem for the interval $(-1,1)$, in terms of the Riesz condition and Hankel forms, are also well known. In part II we give a third solution in terms of a Jacobi-type continued fraction. Again, through an interpretation of the parameters in this continued fraction in terms of "distances" in certain convex bodies and an extension of the work of Karlin and Shapley, the equivalence of the three characterizations is immediate.

\section{STIELTJES-TYPE CONTINUED FRACTIONS AND CONVEX SETS}

1. The monotone Hausdorff moment problem. A sequence of real numbers $\left\{c_{n}\right\}(n=0,1,2, \cdots)$ is called a monotone Hausdorff moment sequence if there exists a monotone nondecreasing real function $\phi(u)$, $0 \leqq u \leqq 1$, such that

$$
c_{n}=\int_{0}^{1} u^{n} d \phi(u), \quad n=0,1,2, \cdots
$$

Received December 8, 1958. The authors wish to acknowledge several helpful suggestions from Professor Walter T. Scott of Northwestern University. 
The problem of determining such a function $\phi(u)$ is known as the monotone Hausdorff moment problem. We shall assume throughout part I unless otherwise designated that $c_{0}=1$.

Wall [3], [4] has shown that a sequence $\left\{c_{n}\right\}$ is a monotone Hausdorff moment sequence if and only if the power series

$$
P(z)=\sum_{n=0}^{\infty} c_{n} z^{n}
$$

has a continued fraction expansion of the form

$$
\frac{1}{1}-\frac{\left(1-g_{0}\right) g_{1} z}{1}-\frac{\left(1-g_{1}\right) g_{2} z}{1}-\cdots
$$

where $0 \leqq g_{p} \leqq 1, p=0,1,2, \cdots$. We shall agree that the continued fraction terminates with the first identically vanishing partial quotient. The sequence $\left\{\left(1-g_{p-1}\right) g_{p}\right\}(p=1,2,3, \cdots)$ is called a chain sequence and the numbers $g_{p}$ are called the parameters of the chain sequence. In general the parameters are not uniquely determined and we designate the minimal set of parameters by $m_{p}$. In this case $m_{0}=0$ and (1.1) takes the form

$$
\frac{1}{1}-\frac{m_{1} z}{1}-\frac{\left(1-m_{1}\right) m_{2} z}{1}-\frac{\left(1-m_{2}\right) m_{3} z}{1}-\cdots
$$

Riesz [2], [1], [3] proved that a sequence $\left\{c_{n}\right\}$ is a monotone Hausdorff moment sequence if and only if the point $\left(c_{1}, c_{2}, \cdots, c_{n}\right), n=1,2,3, \cdots$, is in the convex closure of the arc whose parametric equations are

$$
\begin{aligned}
& x_{1}=t, \\
& x_{2}=t^{2}, \\
& \quad \cdots \\
& x_{n}=t^{n}, 0 \leqq t \leqq 1 .
\end{aligned}
$$

The geometry of these convex bodies is developed rather fully in the work of Karlin and Shapley [1].

2. The connecting theorem. Before stating the theorem which connects continued fractions with convex bodies it is necessary to indicate some special notations for the Hankel determinants. We set

$$
\underline{\Delta}_{2 n}=\left|\begin{array}{ccc}
1 c_{1} & \cdots & c_{n} \\
c_{1} c_{2} & \cdots & c_{n+1} \\
\cdots & \\
c_{n} c_{n+1} & \cdots & c_{2 n}
\end{array}\right|, \quad n=0,1,2, \cdots
$$




$$
\begin{aligned}
& \Delta_{2 n+1}=\left|\begin{array}{cccc}
c_{1} & c_{2} & \cdots & c_{n+1} \\
c_{2} & c_{3} & \cdots & c_{n+2} \\
& \cdots & \\
c_{n+1} & c_{n+2} & \cdots & c_{2 n+1}
\end{array}\right|, \\
& n=0,1,2, \cdots \text {, } \\
& \left(\underline{\Delta}_{-1}=1\right) \text {, }
\end{aligned}
$$

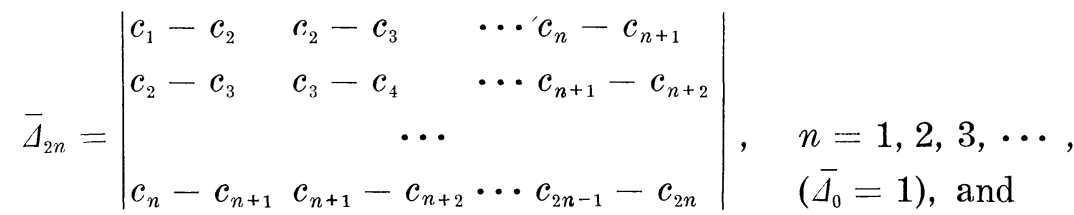

$$
\begin{aligned}
& \bar{\Delta}_{2 n+1}=\left|\begin{array}{cccc}
1-c_{1} & c_{1}-c_{2} & \cdots & c_{n}-c_{n+1} \\
c_{1}-c_{2} & c_{2}-c_{3} \cdot & \cdots & c_{n+1}-c_{n+2} \\
& \cdots & \\
c_{n}-c_{n+1} & c_{n+1}-c_{n+2} \cdots c_{2 n}-c_{2 n+1}
\end{array}\right|, \quad \begin{array}{l}
n=0,1,2, \cdots, \\
\left(\bar{\Delta}_{-1}=1\right) .
\end{array}
\end{aligned}
$$

It is well known that a sequence $\left\{c_{n}\right\}$ is a monotone Hausdorff moment sequence if and only if the Hankel forms

$$
\begin{gathered}
\sum_{i, j=0}^{n} c_{i+j} x_{i} x_{j}, \quad \sum_{i, j=0}^{n} c_{i+j+1} x_{i} x_{j}, \\
\sum_{i, j=0}^{n-1}\left(c_{i+j+1}-c_{i+j+2}\right) x_{i} x_{j}, \quad \sum_{i, j=0}^{n}\left(c_{i+j}-c_{i+j+1}\right) x_{i} x_{j}
\end{gathered}
$$

are all positive semidefinite. In (2.1) replace $c_{2 n}$ by $\underline{c}_{2 n}$, and in (2.2) replace $c_{2 n+1}$ by $\underline{c}_{2 n+1}$. Setting $\underline{\Delta}_{2 n}$ and $\underline{\Delta}_{2 n+1}$ equal to zero, we have the single relation

$$
\underline{c}_{n}=c_{n}-\frac{\underline{\Delta}_{n}}{\underline{\Delta}_{n-2}}, \quad n=1,2,3, \cdots,
$$

provided $\underline{A}_{n-2} \neq 0$. Similarly, (2.3) and (2.4) yield

$$
\bar{c}_{n}=c_{n}+\frac{\bar{\Delta}_{n}}{\overline{\bar{\Delta}}_{n-2}}, \quad n=1,2,3, \cdots,
$$

provided $\bar{\Delta}_{n-2} \neq 0$. If the sequence $\left\{c_{n}\right\}$ is a monotone Hausdorff moment sequence, then the quantities $\underline{c}_{n}$ and $\bar{c}_{n}$ have been interpreted as the "downward" and "upward" projections, respectively, of $c_{n}$ on the boundary of the corresponding convex body [1].

We can now state the following theorem:

THEOREM 2.1. If the sequence $\left\{c_{n}\right\}$ is a monotone Hausdorff moment sequence, then the elements and the minimal parameters in the continued fraction (1.2) can be written in the forms 


$$
a_{n}=\left(1-m_{n-1}\right) m_{n}=\frac{c_{n}-\underline{c}_{n}}{c_{n-1}-\underline{c}_{n-1}}, \quad n=1,2,3, \cdots,\left(\underline{c}_{0}=0\right),
$$

and

$$
m_{n}=\frac{c_{n}-\underline{c}_{n}}{\bar{c}_{n}-\underline{c}_{n}}, \quad 1-m_{n}=\frac{\bar{c}_{n}-c_{n}}{\bar{c}_{n}-\underline{c}_{n}}, \quad n=1,2,3, \cdots .
$$

From the proof it will be clear that a more general theorem is true. If $\left\{c_{n}\right\},\left(c_{0}=1\right)$, is an arbitrary sequence of real numbers and its corresponding Stieltjes-type continued fraction is written in the form (1.2), where no longer it is necessary that $0 \leqq m_{n} \leqq 1, n=1,2,3, \cdots$, the relations (2.7) and (2.8) are still valid.

If $\left\{c_{n}\right\}$ is a monotone Hausdorff moment sequence, then the $m_{n}$ can be interpreted as the ratio of the "distance" of $c_{n}$ to the lower boundary to the "distance" between the upper and lower boundaries of the corresponding convex body. Similar interpretations can be given to the $a_{n}$ and $\left(1-m_{n}\right)$. By Theorem 2.1 the equivalence of the condition in terms of Hankel forms and Wall's characterization in terms of the continued fraction (1.2), for the existence of a monotone Hausdorff moment sequence, is apparent.

Proof. The proof depends upon the following lemma:

Lemma 2.1. The determinants in (2.1), (2.2), (2.3), and (2.4) satisfy the relation

$$
\underline{\Delta}_{k} \bar{\Delta}_{k}=\bar{\Delta}_{k+1} \underline{\Delta}_{k-1}+\underline{\Delta}_{k+1} \bar{\Delta}_{k-1}, \quad k=1,2,3, \cdots .
$$

We shall indicate two proofs to this lemma.

Proof (1). By a substitution and an equivalence transformation, we write the continued fraction (1.2) in the form

$$
\frac{1}{z}-\frac{a_{1}}{1}-\frac{a_{2}}{z}-\frac{a_{3}}{1}-\cdots,
$$

where $a_{k}=\left(1-m_{k-1}\right) m_{k}, \quad k=1,2,3, \cdots, \quad\left(m_{0}=0\right)$. The recurrence formulas for the denominators of the continued fraction (2.10) are given by

$$
B_{2 k}(z)=B_{2 k-1}(z)-a_{2 k-1} B_{2 k-2}(z),
$$

$$
\begin{aligned}
& k=1,2,3, \cdots, \\
& \left(B_{0}(z)=1\right),
\end{aligned}
$$

and

$$
\begin{array}{ll}
B_{2 k+1}(z)=z B_{2 k}(z)-a_{2 k} B_{2 k-1}(z), \quad k=0,1,2, \cdots, \\
& \left(a_{0}=1, B_{-1}(z)=0, B_{0}(z)=1\right) .
\end{array}
$$

Furthermore, we have 


$$
B_{2 k}(z)=\frac{\underline{\underline{A}}_{2 k}(z)}{\underline{1}_{2 k-2}}, \quad k=1,2,3, \cdots
$$

and

$$
B_{2 k+1}(z)=\frac{z \underline{\Delta}_{2 k+1}(z)}{\underline{\Delta}_{2 k-1}}, \quad k=0,1,2, \cdots,
$$

where $\underline{A}_{2 k-2}$ and $\underline{A}_{2 k-1}$ are obtained from (2.1) and (2.2), respectively, and we define

$$
\Lambda_{2 k}(z)=\left|\begin{array}{lllll}
1 & c_{1} & \cdots & c_{k-1} & 1 \\
c_{1} & c_{2} & \cdots & c_{k} & z \\
c_{2} & c_{3} & \cdots & c_{k+1} & z^{2} \\
& & \cdots & \\
c_{k} & c_{k+1} & \cdots & c_{2 k-1} & z^{k}
\end{array}\right|, \quad \quad k=1,2,3, \cdots
$$

and

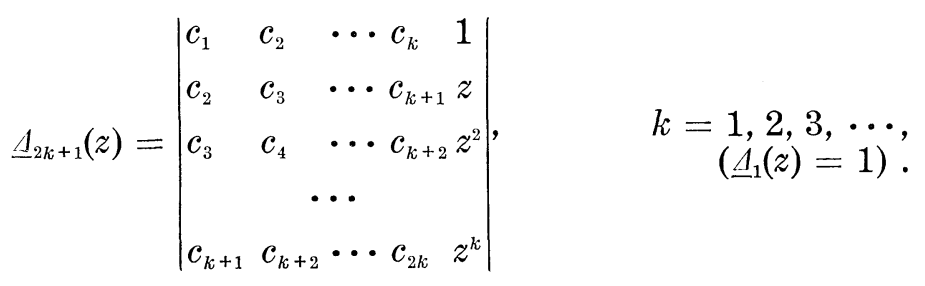

By a sequence of elementary operations on $\underline{A}_{2 k}(z)$ and $\underline{A}_{2 k+1}(z)$ it is seen that $\underline{\Delta}_{k}(1)=\bar{\Delta}_{k-1}, k=1,2,3, \cdots$.

Substituting this result in (2.13) and (2.14) we have

$$
B_{k}(1)=\frac{\bar{\Delta}_{k-1}}{\underline{\Delta}_{k-2}}, \quad k=1,2,3, \cdots .
$$

We also note that

$$
a_{k}=\frac{\underline{\Delta}_{k-3} \underline{\Delta}_{k}}{\underline{\Delta}_{k-2} \underline{\Delta}_{k-1}}, \quad k=1,2,3, \cdots,\left(\underline{\Delta}_{-2}=1\right) .
$$

Substituting the results of (2.17) and (2.18) in (2.11) and (2.12), the relation (2.9) follows immediately.

Proof (2). By Laplace's Development and a sequence of elementary operations, Lemma 2.1 can be established directly. We shall omit the details.

The proof of Theorem 2.1 now follows. Using (2.5) and (2.18), the relation (2.7) is immediate.

The relation (2.8) is established by induction. Assume that 
$0 \leqq m_{n}<1, n=1,2,3, \cdots$. Using (2.5), (2.6), and Lemma 2.1 it is clear that

$$
m_{1}=a_{1}=\frac{c_{1}-\underline{c}_{1}}{c_{0}-\underline{c}_{0}}=\frac{c_{1}-\underline{c}_{1}}{\bar{c}_{1}-\underline{c}_{1}}
$$

where $c_{0}=1$ and we define $\underline{c}_{0}$ to be zero, and $m_{2}=\frac{c_{2}-\underline{c}_{2}}{\bar{c}_{2}-\underline{c}_{2}}$.

Now assume that $m_{k}=\frac{c_{k}-\underline{c}_{k}}{\bar{c}_{k}-\underline{c}_{k}}$. Again using (2.5), (2.6), and Lemma 2.1 in the relation $m_{k+1}=\frac{a_{k+1}}{1-m_{k}}$, the definition for the minimal parameters in a chain sequence [3], the induction is completed. If $m_{k}=1$ then $m_{k+1}$ is defined to be zero. In this case the corresponding moments fall on the upper and lower boundaries of their respective convex bodies.

3. Some results from the theory of chain sequences. Regarding the uniqueness of the parameters $g_{p}$ in the continued fraction (1.1) and the location of the moments in the convex bodies we have the following theorem:

THEOREM 3.1. Given a monotone Hausdorff moment sequence, $\left\{c_{n}\right\}$, let

$$
\lim \frac{c_{k}-\underline{c}_{k}}{\bar{c}_{k}-c_{k}}=q
$$

If $q>1$ the parameters $g_{p}$ in (1.1) are uniquely determined, and if $q<1$ the parameters are not uniquely determined. In case $q=1$ the parameters may or may not be unique.

Proof. Wall [3] proved that the parameters in a chain sequence are uniquely determined if and only if the series

$$
1+\sum_{k=1}^{\infty} \frac{m_{1} m_{2} \cdots m_{k}}{\left(1-m_{1}\right)\left(1-m_{2}\right) \cdots\left(1-m_{k}\right)}
$$

diverges. Making use of this result and Theorem 2.1 our proof is immediate.

We designate the maximal parameters of the chain sequence in the continued fraction (1.1) by $M_{p}$. The maximal parameters can be interpreted in terms of "distances" in the convex bodies by the following theorem:

THEOREM 3.2. The maximal parameters $M_{n}$ in the continued fraction (1.1) can be written in the form 


$$
M_{n}=\frac{c_{n}-c_{n}}{\bar{c}_{n}-\underline{c}_{n}}+\frac{\bar{c}_{n}-c_{n}}{\bar{c}_{n}-\underline{c}_{n}}\left(1 / T_{n}\right), \quad n=1,2,3, \cdots
$$

where

$$
T_{n}=1+\sum_{r=n+1}^{\infty} \frac{c_{n+1}-\underline{c}_{n+1}}{\bar{c}_{n+1}-c_{n+1}} \frac{c_{n+2}-\underline{c}_{n+2}}{\bar{c}_{n+2}-c_{n+2}} \cdots \frac{c_{r}-\underline{c}_{r}}{\bar{c}_{r}-c_{r}}
$$

in the case that the $a_{n+r}, r=1,2,3, \cdots$, of (2.9) are positive. If $a_{n+1}, a_{n+2}, \cdots, a_{n+r}$ are positive, $a_{n+k+1}=0,(k>0)$, and $m_{n+k}<1$, then the summation in (3.3) runs only to $n+k$.

Proof. Wall [3] introduced an expression of the form (3.3) in discussing maximal parameters. Using his results and Theorem 2.1 our proof is immediate.

\section{JACOBI-TYPE CONTINUED FRACTIONS AND CONVEX SETS}

4. The "extended" monotone Hausdorff moment problem. A sequence of real number $\left\{c_{n}\right\}(n=0,1,2, \cdots)$ shall be referred to as an "extended" monotone Hausdorff moment sequence if there exists a monotone nondecreasing real function $\phi(u),-1 \leqq u \leqq 1$, such that

$$
c_{n}=\int_{-1}^{1} u^{n} d \phi(u), \quad n=0,1,2, \cdots .
$$

The problem of determining such a function $\phi(u)$ shall be referred to as the "extended" monotone Hausdorff moment problem. Again we shall assume throughout part II unless otherwise designated that $c_{0}=1$.

The work of Riesz [2] can be applied to the "extended" monotone Hausdorff moment problem. A sequence $\left\{c_{n}\right\}$ is an "extended" monotone Hausdorff moment sequence if and only if the point $\left(c_{1}, c_{2}, \cdots, c_{n}\right), n=$ $1,2,3, \cdots$, is in the convex closure of the are whose parametric equations are given by (1.3) where $-1 \leqq t \leqq 1$.

Let

$$
\frac{1}{b_{1} z+1}-\frac{a_{1} z^{2}}{b_{2} z+1}-\frac{a_{2} z^{2}}{b_{3} z+1}-\cdots
$$

be the Jacobi-type continued fraction expansion of the power series

$$
P(z)=\sum_{n=0}^{\infty} c_{n} z^{n}
$$

We shall agree that the continued fraction terminates with the first identically vanishing partial quotient. We shall show that if the sequence $\left\{c_{n}\right\}$ is an "extended" monotone Hausdorff moment sequence, then the 
$a_{p}$ and $b_{p}$ of (4.1) have the form of a generalized chain sequence and the parameters can again be represented in terms of "distances" in certain convex bodies.

5. The connecting theorem. As in $\S 2$, it is necessary to indicate some special notations for the Hankel determinants corresponding to an "extended" monotone Hausdorff moment sequence. We set

$$
\begin{aligned}
& \Delta_{2 n}=\left|\begin{array}{cccc}
1 & c_{1} & \cdots & c_{n} \\
c_{1} & c_{2} & \cdots & c_{n+1} \\
& & \cdots & \\
c_{n} & c_{n+1} & \cdots & c_{2 n}
\end{array}\right| \\
& n=0,1,2, \cdots \text {, } \\
& \underline{\Delta}_{2 n+1}=\left|\begin{array}{cccc}
1+c_{1} & c_{1}+c_{2} & \cdots c_{n}+c_{n+1} \\
c_{1}+c_{2} & c_{2}+c_{3} & \cdots c_{n+1}+c_{n+2} \\
& \cdots & \\
c_{n}+c_{n+1} & c_{n+1}+c_{n+2} & \cdots & c_{2 n}+c_{2 n+1}
\end{array}\right|, \quad \begin{array}{l}
n=0,1,2, \cdots, \\
\left(\underline{\Delta}_{-1}=1\right),
\end{array} \\
& \bar{\Delta}_{2 n}=\left|\begin{array}{rrrr}
1-c_{2} & c_{1}-c_{3} & \cdots c_{n-1}-c_{n+1} \\
c_{1}-c_{3} & c_{2}-c_{4} & \cdots c_{n}-c_{n+2} \\
& \cdots & \\
c_{n-1}-c_{n+1} c_{n}-c_{n+2} & \cdots c_{2 n-2}-c_{2 n}
\end{array}\right|, \begin{array}{l}
n=1,2,3, \cdots, \\
\left(\bar{J}_{0}=1\right), \text { and }
\end{array} \\
& \bar{\Delta}_{2 n+1}=\left|\begin{array}{cccc}
1-c_{1} & c_{1}-c_{2} & \cdots & c_{n}-c_{n+1} \\
c_{1}-c_{2} & c_{2}-c_{3} & \cdots & c_{n+1}-c_{n+2} \\
& \cdots & \\
c_{n}-c_{n+1} & c_{n+1}-c_{n+2} & \cdots & c_{2 n}-c_{2 n+1}
\end{array}\right|, \begin{array}{l}
n=0,1,2, \cdots, \\
\left(\bar{\Delta}_{-1}=1\right) .
\end{array}
\end{aligned}
$$

The sequence $\left\{c_{n}\right\}$ is an "extended" monotone Hausdorff moment sequence if and only if the Hankel forms

$$
\begin{aligned}
& \sum_{i, j=0}^{n} c_{i+j} x_{i} x_{j}, \quad \sum_{i, j=0}^{n}\left(c_{i+j}+c_{i+j+1}\right) x_{i} x_{j}, \\
& \sum_{i, j=0}^{n-1}\left(c_{i+j}-c_{i+j+2}\right) x_{i} x_{j}, \sum_{i, j=0}^{n}\left(c_{i+j}-c_{i+j+1}\right) x_{i} x_{j}
\end{aligned}
$$

are all positive semidefinite. As in part I replace $c_{2 n}$ by $\underline{c}_{2 n}$ in (5.1) and $c_{2 n+1}$ by $\underline{c}_{2 n+1}$ in (5.2). Setting $\underline{A}_{2 n}$ and $\underline{A}_{2 n+1}$ equal to zero, we have the single relation

$$
\underline{c}_{n}=c_{n}-\frac{\underline{A}_{n}}{\underline{\Delta}_{n-2}}, \quad n=1,2,3, \cdots .
$$

Similarly, (5.3) and (5.4) yield 


$$
\bar{c}_{n}=c_{n}+\frac{\bar{\Delta}_{n}}{\bar{\Delta}_{n-2}}, \quad n=1,2,3, \cdots .
$$

The methods of Karlin and Shapley [1] can be applied so that if the sequence $\left\{c_{n}\right\}$ is an "extended" monotone Hausdorff moment sequence then the quantities $\underline{c}_{n}$ and $\bar{c}_{n}$ of (5.5) and (5.6) are again interpreted as the "downward" and "upward" projections, respectively, of $c_{n}$ on the boundary of the corresponding convex body.

We can now state the following theorem:

THEOREM 5.1. If the sequence $\left\{c_{n}\right\}$ is an "extended" monotone Hausdorff moment sequence, then the elements $a_{n}$ and $b_{n}$ in the continued fraction (4.1) can be written in the forms

$$
\begin{aligned}
& a_{n}=4 m_{n}\left(1-m_{n-1}\right) l_{n}\left(1-l_{n}\right), \quad n=1,2,3, \cdots, \\
& 0 \leqq m_{n} \leqq 1,\left(m_{0}=0\right), 0 \leqq l_{n} \leqq 1, \\
&=\frac{c_{2 n}-\underline{c}_{2 n}}{c_{2 n-2}-\underline{c}_{2 n-2}}, \quad n=1,2,3, \cdots,\left(c_{0}=0\right),
\end{aligned}
$$

where

$$
\begin{aligned}
m_{n} & =\frac{c_{2 n}-\underline{c}_{2 n}}{\bar{c}_{2 n}-\underline{c}_{2 n}}, \quad l_{n}=\frac{c_{2 n-1}-\underline{c}_{2 n-1}}{\bar{c}_{2 n-1}-\underline{c}_{2 n-1}}, \\
1-m_{n} & =\frac{\bar{c}_{2 n}-c_{2 n}}{\bar{c}_{2 n}-\underline{c}_{2 n}}, \quad 1-l_{n}=\frac{\bar{c}_{2 n-1}-c_{2 n-1}}{\bar{c}_{2 n-1}-\underline{c}_{2 n-1}}, n=1,2,3, \cdots,
\end{aligned}
$$

and

$$
\begin{array}{lr}
b_{n}=1-2 m_{n-1}\left(1-l_{n-1}\right)-2\left(1-m_{n-1}\right) l_{n}, & n=1,2,3, \cdots, \\
& \left(l_{0}=m_{0}=0\right), \\
=1-\frac{c_{2 n-1}-\underline{c}_{2 n-1}}{c_{2 n-2}-\underline{c}_{2 n-2}}-\frac{c_{2 n-2}-\underline{c}_{2 n-2}}{c_{2 n-3}-\underline{c}_{2 n-3}}, & n=2,3,4, \cdots .
\end{array}
$$

As in part I it will be clear that a more general theorem is true. If $\left\{c_{n}\right\},\left(c_{0}=1\right)$, is an arbitrary sequence of real numbers and its corresponding Jacobi-type continued fraction (4.1) is written in the form that the $a_{n}$ and $b_{n}$ are given by (5.7) and (5.10), respectively, where $l_{0}=m_{0}=0$ but it is no longer necessary that $0 \leqq l_{n} \leqq 1$ and $0 \leqq m_{n} \leqq 1$, $n=1,2,3, \cdots$, then the relations (5.8) and (5.11) with (5.9) holding are still valid.

If $\left\{c_{n}\right\}$ is an "extended" monotone Hausdorff moment sequence, the geometric interpretations of the $a_{n}, b_{n}, l_{n}$, and $m_{n}$ are apparent.

Proof. The proof depends upon the following lemma.

LEMma 5.1. The determinants in (5.1), (5.2), (5.3), and (5.4) satisfy the relations 


$$
\begin{aligned}
& \underline{\Delta}_{2 k+1} \bar{\Delta}_{2 k+1}=\bar{\Delta}_{2 k+2} \underline{\Delta}_{2 k}+\underline{\Delta}_{2 k+2} \bar{\Delta}_{2 k}, \\
& 2 \underline{\Delta}_{2 k} \bar{\Delta}_{2 k}=\bar{\Delta}_{2 k+1} \underline{\Delta}_{2 k-1}+\underline{\Delta}_{2 k+1} \bar{\Delta}_{2 k-1},
\end{aligned}
$$

Proof. By Laplace's Development and a sequence of elementary operations, Lemma 5.1 can be established directly. We shall omit the details.

The proof to the theorem now follows. A well known formula for the $a_{k}$ is given by

$$
a_{k}=\frac{\underline{\Delta}_{2 k} \underline{\Delta}_{2 k-4}}{\underline{\Delta}_{2 k-2} \underline{\Delta}_{2 k-2}}, \quad k=2,3,4, \cdots,\left(a_{1}=\frac{\underline{\Delta}_{2}}{\underline{\Delta}_{0}^{*}}\right) .
$$

The formulas (5.5) and (5.13) yield (5.8).

By a substitution and an equivalence transformation, we write the continued fraction (4.1) in the form

$$
\frac{1}{b_{1}+z}-\frac{a_{1}}{b_{2}+z}-\frac{a_{2}}{b_{2}+z}-\cdots
$$

The recurrence formula for the denominators of the continued fraction (5.14) is given by

$$
\begin{aligned}
B_{k}(z)= & \left(b_{k}+z\right) B_{k-1}(z)-a_{k-1} B_{k-2}(z), \\
& k=1,2,3, \cdots,\left(a_{0}=1, B_{-1}(z)=0, B_{0}(z)=1\right) .
\end{aligned}
$$

Furthermore, we have

$$
B_{k}(z)=\frac{\underline{A}_{2 k}(z)}{\underline{\Delta}_{2 k-2}}, \quad k=1,2,3, \cdots,
$$

where $\underline{A}_{2 k-2}$ is obtained from (5.1) and we define $\underline{A}_{2 k}(z)$ the same as in (2.15). By a sequence of elementary operations on $\underline{A}_{2 k}(z)$ it is seen that $\underline{\Delta}_{2 k}(-1)=(-1)^{k} \underline{\Lambda}_{2 k-1}, k=1,2,3, \cdots$. Substituting this result in (5.16) we have

$$
B_{k}(-1)=\frac{(-1)^{k} \underline{A}_{2 k-1}}{\underline{A}_{2 k-2}}, \quad k=1,2,3, \cdots .
$$

Setting $z$ equal to -1 in (5.15), using the formulas (5.13) and (5.17), we can solve for $b_{k}$ and obtain (5.11). We note that if we had set $z$ equal to 1 and followed a similar procedure, we would have obtained the formula

$$
b_{n}=\frac{\bar{c}_{2 n-1}-c_{2 n-1}}{c_{2 n-2}-\underline{c}_{2 n-2}}+\frac{c_{2 n-2}-\underline{c}_{2 n-2}}{\bar{c}_{2 n-3}-c_{2 n-3}}-1, \quad n=2,3,4, \cdots .
$$

Assume that $0 \leqq m_{n}<1,0 \leqq l_{n}<1, n=1,2,3, \cdots$ Using (5.5), 
(5.6), and (5.9) it can be shown directly that $b_{1}=1-2 l_{1}$, and $a_{1}=$ $4 m_{1} l_{1}\left(1-l_{1}\right)$. Now by using (5.5), (5.6), (5.9), and Lemma 5.1, (5.11) reduces to $(5.10)$ for $n=k, k=2,3,4, \cdots$. A similar statement applies to (5.8). If $m_{k}=1$ then $m_{k+1}$ is defined to be zero. A similar statement applies to $l_{h}$. In either case the corresponding moments fall on the upper and lower boundaries of their respective convex bodies.

If (5.18) had been used in place of (5.11) we note that (5.10) would have been obtained in the form

$$
\begin{aligned}
b_{n}=2\left(1-l_{n}\right)\left(1-m_{n-1}\right)+2 m_{n-1} l_{n-1}-1, \\
n=1,2,3, \cdots,\left(l_{0}=m_{0}=0\right) .
\end{aligned}
$$

By Theorem 5.1 and the condition in terms of Hankel forms, we can now state a theorem which characterizes the existence of an "extended" monotone Hausdorff moment sequence in terms of continued fractions. This theorem is analogous to Wall's solution [3], [4] for the regular monotone Hausdorff moment sequence. By Theorem 5.1 and an extension of the work of Karlin and Shapley, the equivalence of the continued fraction solution and the condition in terms of Hankel forms, and hence convex bodies, is apparent.

THeOrem 5.2. The sequence $\left\{c_{n}\right\}$ is an "extended" monotone Hausdorff moment sequence if and only if the power series

$$
P(z)=\sum_{n=0}^{\infty} c_{n} z^{n}
$$

has a Jacobi-type continued fraction (4.1) expansion where the $a_{n}$ and $b_{n}$ are given by (5.7) and (5.10), respectively, and $l_{0}=m_{0}=0$, and $0 \leqq l_{n} \leqq 1,0 \leqq m_{n} \leqq 1, n=1,2,3, \cdots$.

It should be pointed out that $P(z)=\sum_{m=0}^{\infty} c_{m} z^{m}$ is a moment generating function for the "extended" monotone Hausdorff moment problem if and only if $Q(w)=(1+z) P(z)$, where $w=\frac{2 z}{1+z}$, is a moment generating function for the regular monotone Hausdorff moment problem. From these relations it is observed that the $l_{n}$ and $m_{n}$ of Theorem 5.1 are equal to $m_{2 n-1}$ and $m_{2 n}, n=1,2,3, \cdots$, respectively, of Theorem 2.1. These results are obtained by contraction.

It can also be noted that $\left\{c_{n}\right\}$ is an "extended" monotone Hausdorff moment sequence if and only if

$$
\left\{d_{n} / 2^{n}\right\}, \quad d_{n}=\sum_{j=0}^{n}\left(\begin{array}{c}
n \\
n-j
\end{array}\right) c_{j},
$$

is a regular monotone Hausdorff moment sequence. This result can be obtained by comparing coefficients in $P(z)$ and $Q(w)$ under the indicated transformation. 
6. The continued fraction of the first differences. We prove the following theorem:

\section{THEOREM 6.1. If}

$$
1+c_{1} z+c_{2} z^{2}+\cdots \sim \frac{1}{b_{1} z+1}-\frac{a_{1} z^{2}}{b_{2} z+1}-\frac{a_{2} z^{2}}{b_{3} z+1}-\cdots,
$$

where

$$
\begin{gathered}
b_{n}=1-2 m_{n-1}\left(1-l_{n-1}\right)-2\left(1-m_{n-1}\right) l_{n}, n=1,2,3, \cdots, \\
a_{n}=4 m_{n}\left(1-m_{n-1}\right) l_{n}\left(1-l_{n}\right), n=1,2,3, \cdots,\left(l_{0}=m_{0}=0\right),
\end{gathered}
$$

then

$$
\Delta c_{0}+\Delta c_{1} z+\Delta c_{2} z^{2}+\cdots \sim \frac{a_{0}^{*}}{b_{1}^{*} z+1}-\frac{a_{1}^{*} z^{2}}{b_{2}^{*} z+1}-\frac{a_{2}^{*} z^{2}}{b_{3}^{*} z+1}-\cdots,
$$

where $\Delta c_{n}=c_{n+1}-c_{n}, n=1,2,3, \cdots,\left(\Delta c_{0}=1-c_{1}\right)$, and

$$
\begin{aligned}
& b_{1}^{*}=1-2 l_{1}\left(1-m_{1}\right) \text {, } \\
& b_{n}^{*}=1-2 m_{n-1}\left(1-l_{n}\right)-2 l_{n}\left(1-m_{n}\right), \quad n=2,3,4, \cdots \text {, } \\
& a_{0}^{*}=2\left(1-l_{1}\right) \text {, } \\
& a_{n}^{*}=4 l_{n}\left(1-l_{n+1}\right) m_{n}\left(1-m_{n}\right) \text {, } \\
& n=1,2,3, \cdots \text {. }
\end{aligned}
$$

Proof. In order to prove the theorem it is necessary to note some determinants for the sequence $\left\{\Delta c_{n}\right\}$ corresponding to $\underline{\Delta}_{2 n}$ and $\underline{A}_{2 n+1}$ of (5.1) and (5.2), respectively, for the sequence $\left\{c_{n}\right\}$. Noting (5.3) and (5.4) we observe that

$$
\underline{\Delta}_{2 k}^{*}=\bar{\Delta}_{2 k+1}, \underline{\Delta}_{2 k+1}^{*}=\bar{\Delta}_{2 k+2}, \quad k=0,1,2, \cdots .
$$

We observe directly that

$$
a_{0}^{*}=1-c_{1}=\Delta c_{1}=2\left(1-l_{1}\right) .
$$

Using (5.13) and (6.7) we note that

$$
a_{k}^{*}=\frac{\underline{\Delta}_{2 k}^{*} \underline{\Delta}_{2 k-4}^{*}}{\underline{\Delta}_{2 k-2}^{*} \underline{\Delta}_{2 k-2}^{*}}=\frac{\bar{\Delta}_{2 k+1} \Delta_{2 k-3}}{\bar{\Delta}_{2 k-1} \bar{\Delta}_{2 k-1}} .
$$

The relations in (6.6) can now be established by (5.5) (5.6), (5.9), and Lemma 5.1.

Now, by (5.5), (5.10), (5.11), and (6.7),

$$
b_{k}^{*}=1-\frac{\underline{\Delta}_{2 k-1}^{*} \underline{\Delta}_{2 k-4}^{*}}{\underline{\Delta}_{2 k-2}^{*} \underline{\Delta}_{2 k-3}^{*}}-\frac{\underline{\Delta}_{2 k-2}^{*} \underline{\Delta}_{k-5}^{*}}{\underline{\Delta}_{2 k-3}^{*} \underline{\Delta}_{2 k-4}^{*}}
$$




$$
\begin{aligned}
& =1-\frac{\bar{\Delta}_{2 k} \bar{\Delta}_{2 k-3}}{\bar{\Delta}_{2 k-1} \bar{\Delta}_{2 k-2}}-\frac{\bar{\Delta}_{2 k-1} \bar{\Delta}_{2 k-4}}{\bar{\Delta}_{2 k-2} \bar{\Delta}_{2 k-3}}, \\
& \quad \quad k=1,2,3, \cdots,\left(\underline{\Delta}_{-1}^{*}=\underline{A}_{-2}^{*}=1, \underline{\Delta}_{-3}^{*}=0\right) .
\end{aligned}
$$

Now again by (5.5), (5.6), (5.9), and Lemma 5.1, the relations in (6.5) follow.

We note that a similar proof could be given for the corresponding theorem for a regular monotone Hausdorff moment sequence, thereby giving another proof to this well known result [4].

\section{REFERENCES}

1. S. Karlin and L. S. Shapley, Geometry of moment spaces, Mem. Amer. Math. Soc. no. 12 (1953).

2. F. Riesz, Sur certain systems singuliérs d'equations intégrales, Ann. de l'Éc. Nor., (3) 28 (1911), 34-62.

3. H. S. Wall, Analytic theory of continued fractions, Van Nostrand, 1948.

4. H. S. Wall, Continued fractions and totally monotone sequences, Trans. Amer. Math. Soc., 48 (1940), 165-184.

The Ohio State UnIVERSity

DENISON UNIVERSITY 



\section{PACIFIC JOURNAL OF MATHEMATICS}

\section{EDITORS}

\section{David Gilbarg}

Stanford University Stanford, California

\section{R. A. Beaumont}

University of Washington

Seattle 5, Washington

\section{A. L. Whiteman}

University of Southern California Los Angeles 7, California

L. J. Paige

University of California

Los Angeles 24, California

\section{ASSOCIATE EDITORS}

E. F. BECKENBACH
C. E. BURGESS
E. HEWITT
A. HORN

A. HORN

\author{
V. GANAPATHY IYER \\ R. D. JAMES \\ M. S. KNEBELMAN \\ L. NACHBIN
}

I. NIVEN

T. G. OSTROM

H. L. ROYDEN

M. M. SCHIFFER
E. G. STRAUS

G. SZEKERES

F. WOLF

K. YOSIDA

\section{SUPPORTING INSTITUTIONS}

\author{
UNIVERSITY OF BRITISH COLUMBIA \\ CALIFORNIA INSTITUTE OF TECHNOLOGY \\ UNIVERSITY OF CALIFORNIA \\ MONTANA STATE UNIVERSITY \\ UNIVERSITY OF NEVADA \\ OREGON STATE COLLEGE \\ UNIVERSITY OF OREGON \\ OSAKA UNIVERSITY \\ UNIVERSITY OF SOUTHERN CALIFORNIA
}

\author{
STANFORD UNIVERSITY \\ UNIVERSITY OF TOKYO \\ UNIVERSITY OF UTAH \\ WASHINGTON STATE COLLEGE \\ UNIVERSITY OF WASHINGTON \\ * * * * \\ AMERICAN MATHEMATICAL SOCIETY \\ CALIFORNIA RESEARCH CORPORATION \\ HUGHES AIRCRAFT COMPANY \\ SPACE TECHNOLOGY LABORATORIES
}

Mathematical papers intended for publication in the Pacific Journal of Mathematics should be typewritten (double spaced), and the author should keep a complete copy. Manuscripts may be sent to any one of the four editors. All other communications to the editors should be addressed to the managing editor, L. J. Paige at the University of California, Los Angeles 24, California.

50 reprints per author of each article are furnished free of charge; additional copies may be obtained at cost in multiples of 50 .

The Pacific Journal of Mathematics is published quarterly, in March, June, September, and December. The price per volume (4 numbers) is $\$ 12.00$; single issues, $\$ 3.50$. Back numbers are available. Special price to individual faculty members of supporting institutions and to individual members of the American Mathematical Society: $\$ 4.00$ per volume; single issues, $\$ 1.25$.

Subscriptions, orders for back numbers, and changes of address should be sent to Pacific Journal of Mathematics, 2120 Oxford Street, Berkeley 4, California.

Printed at Kokusai Bunken Insatsusha (International Academic Printing Co., Ltd.), No. 6, 2-chome, Fujimi-cho, Chiyoda-ku, Tokyo, Japan.

PUBLISHED BY PACIFIC IOURNAL OF MATHEMATICS, A NON-PROFIT CORPORATION

The Supporting Institutions listed above contribute to the cost of publication of this Journal, but they are not owners or publishers and have no responsibility for its content or policies. 


\section{Pacific Journal of Mathematics}

\section{Vol. 9, No. $3 \quad$ July, 1959}

Errett Albert Bishop, A minimal boundary for function algebras . . . . . . . . . . . . 629

John W. Brace, The topology of almost uniform convergence . . . . . . . . . . . . 643

Cecil Edmund Burgess, Chainable continua and indecomposability .......... 653

L. Carlitz, Multiplication formulas for products of Bernoulli and Euler

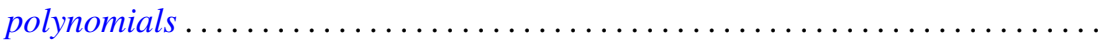

Eckford Cohen, A class of residue systems (mod $r$ ) and related arithmetical

functions. II. Higher dimensional analogues ....................

Shaul Foguel, Boolean algebras of projections of finite multiplicity . . . . . . . . . .

Richard Robinson Goldberg, Averages of Fourier coefficients .................

Seymour Goldberg, Ranges and inverses of perturbed linear operators .

Philip Hartman, On functions representable as a difference of convex functions ....

Milton Vernon Johns, Jr. and Ronald Pyke, On conditional expectation and

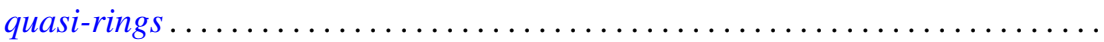

Robert Jacob Koch, Arcs in partially ordered spaces ....................

Gregers Louis Krabbe, A space of multipliers of type $L^{p}(-\infty, \infty) \ldots \ldots \ldots \ldots$

John W. Lamperti and Patrick Colonel Suppes, Chains of infinite order and their

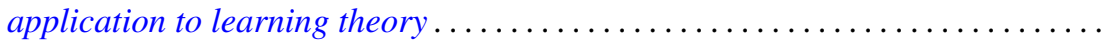

Edith Hirsch Luchins, On radicals and continuity of homomorphisms into Banach

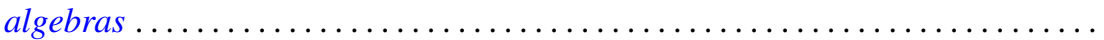

T. M. MacRobert, Multiplication formulae for the E-functions functions of their parameters.

Michael Bahir Maschler, Classes of minimal and representative domains and their kernel functions.

William Schumacher Massey, On the imbeddability of the real projective spaces in Euclidean space.

Thomas Wilson Mullikin, Semi-groups of class $\left(C_{0}\right)$ in $L_{p}$ determined by parabolic differential equations

Steven Orey, Recurrent Markov chains

Ernest Tilden Parker, On quadruply transitive groups ........ . .

Calvin R. Putnam, On Toeplitz matrices, absolute continuity, and unitary

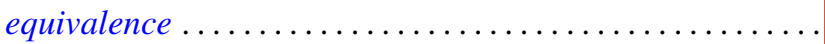

Helmut Heinrich Schaefer, On nonlinear positive operators.

Robert Seall and Marion Wetzel, Some connections between continued fractions and convex sets

Robert Steinberg, Variations on a theme of Chevalley

Olga Taussky and Hans Zassenhaus, On the similarity transformation between a

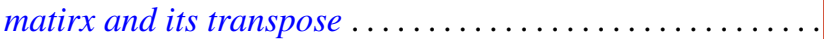

Emery Thomas, The suspension of the generalized Pontrjagin cohomology

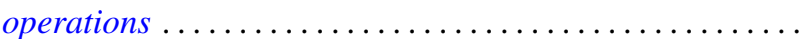

Joseph L. Ullman, On Tchebycheff polynomials ..................... 913

Richard Steven Varga, Orderings of the successive overrelaxation scheme ........ 925

Orlando Eugenio Villamayor, Sr., On weak dimension of algebras . 